\title{
Un trastorno psicológico llamado TRAPSIPO
}

\author{
A psychological disorder called TRAPSIPO
}

\section{Luis Hesneide Morales-López ${ }^{1, a}$}

\section{RESUMEN}

Este artículo postula la existencia de un trastorno psicológico que se presenta en ciertas personas que viven en condiciones de precariedad económica. Un trastorno que es posible denominarlo con la sigla TRAPSIPO (Trastorno Psicológico de la Pobreza) y que, como cualquier otra entidad nosológica, tiene sus signos y síntomas, cuyas características principales son la desesperanza, la baja autoestima, la apatía, el negativismo, el pesimismo, entre otros. Se analizan sus diversos factores y se plantean criterios de diagnóstico diferencial frente a los denominados trastornos depresivos. Además, se analizan las implicaciones sociales del reconocimiento de este trastorno.

PALABRAS CLAVE: trastorno, diagnóstico diferencial, psicología, pobreza, políticas públicas.

\section{SUMMARY}

This article argues that the TRAPSIPO (by its initials in Spanish) is a psychological disorder in certain people living in poverty. A disorder that, like any other, has its signs and symptoms such as: hopelessness, low self-esteem, apathy, negativism and pesimism, among others. Its multiple factors are analyzed and also criteria for differential diagnosis with the depressive disorders are established. Further, social implications regarding to acknowledging this disorder are discussed.

KEYWORDS: disorder, differential diagnosis, psychology, poverty, public policies.

\section{INTRODUCCIÓN}

Considero que tres son los factores principales, además de la frecuente interrelación que he tenido con quienes viven con el mínimo para subsistir -sobre todo en los últimos años con motivos de mi tesis doctorallos que me han llevado a plantear el Trastorno Psicológico de la Pobreza (TRAPSIPO) como una nueva entidad nosológica.

El primer factor descansa sobre la trayectoria y los aportes del Dr. Reynaldo Alarcón Napurí, en el área de la Psicología de la Pobreza, quien, al respecto refiere: La pobreza es algo más que un estado de depresión económica y una forma de injusticia social. Es un poderoso factor que influye sobre la conducta de los individuos, estableciendo un patrón modal de vida entre la gente que se encuentra sometida a ella. Genera sistema de valores, actitudes, estilos de pensar, sentir, reaccionar, formas de conducta más o menos uniformes que pone a los grupos pobres en franca diferencia en cuanto a su comportamiento, de los individuos de los estratos socioeconómicos medios y elevados. (Alarcón, 1986, p.98)

El segundo es el relacionado al enfoque sociológico de la pobreza propuesto por Oscar Lewis, quien acuñó el concepto "cultura de la pobreza" haciendo alusión a la cotidianidad de diferentes personas y familias que viven esta condición (Lewis, 1972). Al respecto, a pesar del valor descriptivo de su caracterización y de señalar que la cultura de la pobreza surge como

Unidad de Investigación, Consultora Despertares LHML. Chimbote, Perú.

a Doctor en Psicología por la Universidad Peruana Cayetano Heredia. Psicólogo por la Universidad Nacional Mayor de San Marcos. Director General de Consultora Despertares LHML ORCID: https://orcid.org/0000-0003-0957-2577 
consecuencia de vivir en entornos socioeconómicos desfavorables, lo que es resultante de sociedades desiguales e inequitativas, puede decirse que su aporte, a juzgar por las descripciones que hace de las familias, contiene atisbos de la idea de que los pobres, al estar inmersos en esta cultura, son, de alguna manera, responsables de esta situación. Frente a tal conclusión, sería posible culpar a los pobres por permanecer en esta condición de vida.

El tercer factor es el relacionado con la cada vez más abundante literatura en torno al impacto que las carencias sensoriales o sociales durante los primeros años de vida tienen sobre el cerebro. Es decir, la evidencia de esta nueva entidad nosológica descansa en hallazgos sólidos, tangibles, de que vivir en condiciones de pobreza tiene su correlato anatómico y fisiológico en diversas zonas del cerebro de las personas que están inmersas en estas condiciones de precariedad (Kandel, 2005; Merz et al., 2019). $\mathrm{Al}$ respecto, Babcock (2014) señala que la pobreza, condición asociada a elevados niveles de estrés, afecta el desarrollo cerebral. Por su parte, Mathewson (2017) afirma que cuando se vive en pobreza el sistema límbico constantemente envía mensajes estresores y de temor al córtex prefrontal, al que sobrecargan impidiendo el desarrollo de habilidades de solución de problemas, de fijar metas y de completar tareas de manera eficiente. La exposición a constantes eventos estresores y a continuas situaciones de vulnerabilidad genera modificaciones en el cerebro. Asimismo, Rocheleau (2019), afirma que las personas de escasos recursos económicos tienen menor volumen de tejido celular en las áreas del hipocampo y en las del lóbulo frontal y temporal, las cuales son zonas importantes para las funciones de la memoria y el pensamiento complejo, tales como el razonamiento y la toma de decisiones.

Cabe enfatizar que el devenir de la nomenclatura nosológica descansó siempre en alguien con "ojo clínico", lo que permitió reconocer tal o cual trastorno que, de otra forma, se habría mantenido ignorado durante largo tiempo. Tal es el caso, por ejemplo, del Trastorno Negativista Desafiante, que por primera vez apareció como trastorno en 1980 en el DSMIII. Se consideraba tan sólo como característica de un mal comportamiento de todo aquel niño y adolescente que presentaba conductas negativistas, desafiantes y desobedientes y comportamiento hostil hacia las figuras de autoridad. Ahora es ampliamente aceptado de que se trata de un trastorno, aunque los cuestionamientos sobre sus síntomas, severidad y la frecuencia con la que aparece han sido una constante. La propuesta del TRAPSIPO como una nueva entidad nosológica seguirá probablemente la misma dirección, la de despertar ciertos cuestionamientos, por ello es válido referirse con detalle a sus signos y síntomas, así como a los factores que estarían detrás de su aparición y su posible tratamiento.

Al respecto, es característico en aquellos individuos con TRAPSIPO haber perdido toda esperanza de progresar y de no poder hacer nada para solucionar las dificultades que se presentan en la vida. La actitud derrotista, la apatía, la desidia, la renuncia a un mejor porvenir, la conformidad con la situación en la que se vive y la actitud resignada de no ver alternativa de cambio de los acontecimientos adversos son típicos en ellos. También los caracteriza la inclinación a caer en los vicios, no planear para el futuro, no tener la tendencia a ahorrar, considerar que la vida no tiene sentido y que es mejor vivir el día a día a causa de que el futuro es incierto. Además, la aversión a tomar riesgos.

Es importante enfatizar que junto a la pobreza, que es el principal factor que desencadena el TRAPSIPO, existen otros factores que estarían potenciando el surgimiento de este trastorno, entre los cuales están el pertenecer a una familia sin cohesión, sin organización y sin reglas y/o con alto nivel de conflicto entre los miembros; haber sufrido abandono en la niñez o haber estado en situación de orfandad, haber sido víctima de explotación infantil y, en algunos casos, haber tenido padres alcohólicos.

Vale señalar que la pobreza se define, siguiendo a Sen (2000), como el sinnúmero de experiencias y condiciones que menoscaban la posibilidad de desplegar todas las potencialidades que tiene la condición humana y que priva a la persona de una existencia prolongada y sana, productiva y creativa, así como de poder disfrutar de una adecuada condición de vida, de una plena libertad, dignidad y respeto por sí mismo y de los demás.

Considerando que la categoría diagnóstica que aquí se propone como TRAPSIPO es un desorden que compromete básicamente la psiquis de las personas, es de suma utilidad llevar a cabo un diagnóstico diferencial con los denominados trastornos depresivos, que según la Organización Mundial de la Salud (2019) forman parte de los trastornos del estado de ánimo, toda vez que existe la posibilidad de que se pueda concebir que el TRAPSIPO está dentro de estos 
trastornos y que éste no representa una entidad nueva. El delimitar sus manifestaciones permitirá dilucidar cualquier confusión o imprecisión.

Al respecto de los trastornos depresivos, y específicamente del trastorno depresivo recurrente, la Organización Mundial de la Salud (2019) a través del CIE-11, indica que éste se caracteriza principalmente por antecedentes de al menos dos episodios depresivos en los que han sido frecuentes un estado de ánimo casi a diario deprimido o disminución del interés en las actividades durante al menos dos semanas, acompañado de otros síntomas como dificultad para concentrarse, sentimientos de inutilidad o culpa excesiva o inapropiada, desesperanza, pensamientos recurrentes de muerte o suicidio, cambios en el apetito o el sueño, agitación o enlentecimiento psicomotor y energía reducida o fatiga. Además, en aras de un mejor diagnóstico diferencial, también es necesario distinguir el TRAPSIPO con el trastorno distímico, el cual se caracteriza, según la Organización Mundial de la Salud (2019), por un estado de ánimo depresivo persistente (que dura dos años o más) que se acompaña de otros síntomas como interés o placer notablemente disminuidos en las actividades, concentración y atención reducidas o indecisión, autoestima baja o culpa excesiva o inapropiada, desesperanza sobre el futuro, sueño perturbado o aumento del sueño, disminución o aumento del apetito y energía baja o fatiga.

El detallar los signos y síntomas de los trastornos mencionados es de suma conveniencia dado que permite señalar con objetividad que el TRAPSIPO es en realidad una nueva categoría nosológica. Si bien algunos de los síntomas del TRAPSIPO son también propios de estos trastornos depresivos; tales como los sentimientos de inutilidad, desesperanza, autoestima baja y desesperanza sobre el futuro, también es cierto que muchos de los aspectos centrales de estos diagnósticos no tienen alcance para el TRAPSIPO, tales como la culpa excesiva o inapropiada, pensamientos recurrentes de muerte o suicidio, cambios en el apetito o el sueño, agitación o enlentecimiento psicomotor, sueño perturbado o aumento del sueño, disminución o aumento del apetito, energía baja o fatiga, etc.

Se considera que el trastorno que aquí se postula con la denominación de TRAPSIPO no es nuevo ni producto de nuestros tiempos, podría decirse que ha existido siempre en ciertas personas que viven en condiciones de pobreza económica durante prolongado tiempo. El asunto es que no se le ha considerado como tal, siempre ha estado entremezclado con otros diagnósticos o simplemente se le ha concebido como conductas individuales típicas de personas que viven en condiciones de precariedad. El tan extendido concepto de "cultura de la pobreza" de Oscar Lewis, quizá haya contribuido a que se le considere como un aspecto "normal" del comportamiento y de que estos rasgos son "culturales" pertenecientes a un colectivo que vive en esta condición.

Por otro lado, es conveniente precisar que no todas las personas que viven en condiciones de precariedad económica y que presencian los otros factores asociados desarrollan el TRAPSIPO. Es más, hay algunos que viviendo en estas condiciones adversas mantienen la esperanza que a través del esfuerzo y del sacrificio es posible vivir en mejores condiciones de vida. Es decir, hay quienes a pesar de vivir en condiciones desfavorables, a pesar de experimentar grandes vicisitudes, mantienen incólumes su fe y su esperanza y éstas se evidencian en acciones tangibles como el ahorrar gradualmente, el involucrarse en hábitos saludables, el motivar a los hijos a estudiar y seguir esforzándose día a día, etc.

Consideramos que el anterior razonamiento se da en toda categoría nosológica, independiente de que sea esta un trastorno o una enfermedad. Es decir, no necesariamente los factores de riesgo significan causalidad. En este sentido, en el caso de la depresión, por ejemplo, estar inmerso en sus factores de riesgo, tales como esquemas cognitivos disfuncionales, eventos de vida estresantes, antecedentes de depresión en los padres, dificultades en las relaciones personales, etc. (Hammen, 2005), no necesariamente desencadena este trastorno. Tan similar como ocurre, por ejemplo, con los factores de riesgo para la diabetes: sobrepeso, falta de actividad física, hábito de fumar, el frecuente consumo de bebidas azucaradas, el excesivo consumo de carbohidratos en la dieta alimenticia, entre otros. No todos los que están expuestos a estos factores de riesgo desarrollan la enfermedad (American Diabetes Association, s/f).

Vale acotar que los que sufren de TRAPSIPO no necesariamente presentan todas las características señaladas dentro de su sintomatología. Es decir, cabe la posibilidad de sufrir este trastorno sin que, por ejemplo, se manifieste una tendencia a caer en los vicios o sin que se exprese un miedo extremo a tomar riesgos. En realidad, en toda categoría diagnóstica es posible identificar características que no se presentan en todos los casos de la misma. Una evidencia clara 
de tal razonamiento es lo que ocurre, por ejemplo, con las personas que sufren de diabetes; no todos ellos presentan todos los signos y síntomas: mucha sed (polidipsia), la sensación de mucha hambre (polifagia), la necesidad de orinar continuamente (poliuria), la pérdida de peso, el cansancio, la visión borrosa, el hormigueo o entumecimiento de manos y pies y las infecciones fúngicas en la piel recurrentes (Organización Mundial de la Salud, 2018).

Entonces, haciendo esta salvedad, las personas que sufren de este TRAPSIPO representarían básicamente, parafraseando a Bauman (2003), "vidas desperdiciadas" en quienes es común la sensación de infortunio y desesperanza y que ya no es posible mejorar las condiciones de existencia. En quienes sufren el TRAPSIPO pareciera que la vida de prolongado sufrimiento ha impactado la estructura psíquica y se evidencia el fatalismo, el pesimismo, el sentimiento de desvalorización, de pobre autoconcepto, etc.

"Ellos tienen todo lo que uno debe tener en casa. Cómo quisiera tener lo que ellos tienen. Y a veces viene lo peor, te das cuenta, ves la realidad, cosa que no vas a llegar a tener, porque no gano suficiente, gano diez soles, gano cinco, a veces gano veinte, cómo puedo tener lo que ellos tienen. Nunca la vida voy a llegar a tener. Ellos tienen y yo no [...] te pones a pensar que vivir tranquilo, asi seas humilde, el trabajo más humilde, asi sea botando basura, recogiendo fierros, lo que sea, estás trabajando, es humilde, no ganas suficiente, por lo menos sale para que puedas llevar un pan a tu casa. Y creo que para mí es suficiente. Ellos tienen muchas cosas y yo me respondo entre yo mismo. Quisiera tenerlo pero no puedo. La situación es fea y es bien complicada. Y pienso que, con vivir tranquilo sin hacer daño a nadie, sin buscar problemas a nadie, así tengas un pan que comer, estoy feliz [...]Trabajo, para qué, sí trabajo.....pero, yo creo que de acá lo que viene es lo peor porque quizás los huesos no me van a responder como debe de ser, no me van a responder y no quiero ser una carga para ellos". Varón de 35 años, Chimbote (Morales, 2020).

"[...] no hay ayuda no, no hay de parte de la alcaldía, no hay mucha ayuda, a la vez nos quedamos olvidados, en el campo... por eso, tristes nomás crecemos, por eso hasta ahorita nomás hasta donde Dios nos dé para vivir, ahí nomás estaremos que vamos hacer pe', no hay otra ayuda". Varón de 69 años, Juliaca, Puno (Morales, 2020).

"Tengo una niñita de seis años [...]muy despierta, me dice: 'Yo quiero ser una ingeniera' me dice y me hace unas preguntas que me hacen llorar, ¿no?. Ella me dice, 'mamá, te quiero decir algo, yo sé, yo sé leer mucho' dice, 'yo estoy aprendiendo todas mis tareas, estoy aprendiendo todo pero te hago una pregunta. Si mi hermana también estudió, acabó sus estudios qué es ahora. Ella no es nada, no estudió más'. Ella me reclama por su hermana la mayor. Porque mi hija la mayor sí terminó su secundaria y ella me dice, 'qué ha estudiado ella', me dice. Bueno, le digo, no hubo para darle más estudios. Y me dice. 'Para mí habrá?. ¿Hay para mí? Porque yo quiero estudiar, quiero ser una ingeniera'. Sí, le digo, para ti sí se va a poder le digo. Yo me voy a trabajar le digo. Porque uno de ustedes tiene que ser algo en la vida, le digo. Cuando yo esté más viejita, tú me vas a ver a mí le digo. Con tu profesión, yo ya no voy a trabajar, tú me vas a ver a mí, le digo. 'Ah ya' dice. 'Entonces yo le voy echar más fuerzas a mis estudios', me dice. 'Porque yo creo en tu palabra', me dice. $Y$ a veces, todo sus palabras me hace llorar, me hace pensar. Su papá también piensa ¿no?, y también llora. Él le dice, sí hijita, nosotros vamos hacer un gran sacrificio por ti, porque por tus otros hermanos no lo hemos hecho. Tú vas a seguir estudiando hijita, le dice. Pienso que mi hija, ahorita, no nos entiende porque es chiquita ¿no?, porque es una pequeña, pero de repente de aquí a unos cinco años así, cuando ella ya sea mayorcita tengo que hacerle entender que yo no tengo la posibilidad. No le daré lo que ella quiere, porque usted sabe para que sea ingeniera me parece que son gastos, que se yo, ¿no?. Para que sea en una universidad me parece que es un gasto y como ella no entiende yo le comento asía mi hija". GF Mujeres, mayores de 50 años, Chimbote (Morales, 2020).

Los testimonios grafican una característica central del TRAPSIPO; la desesperanza aprendida, definida como la percepción de que los eventos futuros no se pueden evitar, que no existen esperanzas de cambio y que es imposible escapar del destino (Ardila, 1979). Además, el organismo aprende a no responder, dado que la acción no ocasiona cambio alguno, por consiguiente se genera la expectativa de que en el futuro tampoco habrá relación de contingencia entre las acciones y las consecuencias (Seligman, 1975).

Al respecto de esta característica del TRAPSIPO, Medina y Florido (2005) señalan que la renuncia a un mejor porvenir, la conformidad con la situación en la que se vive, así como la actitud resignada de no ver alternativa de cambio de los acontecimientos adversos contribuyen a que el pobre fundamente su inclinación 
a vivir el día a día, a caer en los vicios o a no planear para el futuro o a no tener la tendencia a ahorrar. $\mathrm{Y}$ en este sentido Sartre aporta al entendimiento de este aspecto central del TRAPSIPO: "más vale la resignación que una esperanza constantemente frustrada"; porque, según De Gaulejac (2015), el costo psíquico de la resignación es menos elevado que la desesperanza de querer que las cosas cambien cuando no van a cambiar. También Rodríguez Rabanal (1989) estaría contribuyendo a entender el TRAPSIPO en su libro Cicatrices de la pobreza, en el que somete a consideración el cómo la exclusión deja sus huellas imperecederas en el ser humano que es apartado de la sociedad. Es decir, el diario padecer por vivir en condiciones extremas, que es algo real y concreto, se convierte en estructura psíquica. En otras palabras, en el TRAPSIPO la miseria material tiene su correlato, va de la mano, con la miseria psíquica.

Todo lo anterior respecto a esta nueva propuesta nosológica, en la que la miseria absoluta destruye el potencial psíquico humano que imposibilita la aparición de la resiliencia, es condensado por Emerson, quien al respecto refiere que lo malo conduce a lo malo, la miseria lleva a la miseria, y los pobres seguirán siendo cada vez más pobres. A no ser que se haga algo para que ello no ocurra (Lera, 2009).

En aras de un mejor entendimiento del TRAPSIPO, nuevamente nos valemos de la diabetes. Al respecto, se sabe que en ella están presentes factores de riesgo hereditarios y ambientales. Entre los factores externos: sobrepeso, falta de actividad física, hábito de fumar, entre otros. Cabría preguntarse: ¿Todos los que están expuestos a estos factores de riesgo desarrollan la enfermedad? La realidad indica que no necesariamente. Por su parte, en el trastorno de la TRAPSIPO indicábamos los siguientes factores de riesgo: pobreza, abandono físico y moral, falta de cuidados parentales, conflictos familiares, falta de apego, padres alcohólicos, embarazo adolescente, trabajo infantil, deserción escolar, etc. Siguiendo la misma lógica anterior: ¿Todos los que están expuestos a vivir en condiciones de pobreza, al abandono físico y falta de cuidados parentales, al trabajo infantil, entre otros factores, desarrollan el TRAPSIPO? La respuesta también es no, un no contundente.

Aquí vale analizar con mayor profundidad en los factores de riesgo. Nos damos cuenta, plenamente, que, en el caso de la diabetes, los factores de riesgo fácilmente podrían evitarse. Basta dos interrogantes para sostener este argumento: ¿Acaso no se puede evitar el consumo de bebidas azucaradas? ¿Acaso es imposible hacer ejercicios físicos y evitar el sedentarismo? La respuesta es obvia para darse cuenta que este argumento tiene sentido. Las respuestas dan pie a la afirmación de que la diabetes podría evitarse $\mathrm{o}$, en todo caso, disminuir su tasa de ocurrencia. Entonces, ¿por qué no se toman acciones para no caer en esta enfermedad? ¿Por qué tanta su incidencia/ prevalencia si se podría evitarla cambiando los hábitos de vida?

Ahora veamos lo concerniente al principal factor de riesgo de este trastorno; la pobreza. ¿Está en la posibilidad de una persona; niño y/o adolescente el llevar una vida alejada de la pobreza y miseria si es que se nace dentro de una familia que está sumida en esta condición? La respuesta seria no; un no categórico. Pero consideremos también aquellos otros factores que muchas veces coexisten con el factor principal, potenciando el surgimiento de este trastorno. ¿Está en la posibilidad de una persona; niño y/o adolescente el evitar los conflictos familiares? ¿Está en la posibilidad de una persona; niño y/o adolescente evitar el alcoholismo de sus padres? ¿Y qué hay de la posibilidad de evitar ser víctima de abandono y cuidados parentales? ¿Y habrá la posibilidad de evitar el trabajo a temprana edad? Y las interrogantes podrían seguir. La respuesta a todas ellas, definitivamente serían no, un no categórico. Es decir, queda absolutamente claro que en el caso del TRAPSIPO, el factor de riesgo principal y los factores de riesgo asociados son menos controlables. En otras palabras, no hay independencia del sujeto, simplemente se está inmerso en estos condicionantes y la posibilidad de evadir su influjo es mínima o inexistente.

Sin embargo, a pesar de la imposibilidad de evitar los factores de riesgo del TRAPSIPO es bastante evidente que existe en el colectivo la idea de que, en cierto grado, los que lo padecen, lo desean. Por ello es fácil escuchar frases como: "son dejados", "son perezosos", "son conformistas", "no se esfuerzan porque así son, porque quieren ser así", etc., y así un sinfín de adjetivos y frases que llevan consigo atribución de causalidad personal, individual o interna de los síntomas del TRAPSIPO.

$\mathrm{Y}$ al hurgar con mayor detalle, pensamos que esta atribución de causalidad individual o personal del TRAPSIPO descansa en el hecho de que existen casos extraordinarios de personas resilientes que no han desarrollado este trastorno a pesar de haber estado expuestas a condiciones de pobreza extrema. De allí, 
creemos que nace todo argumento de atribución causal del TRAPSIPO. Es decir, los casos emblemáticos de seres humanos de enorme resiliencia, que no han desarrollado el trastorno y que, por el contrario, se mantienen optimistas, con esperanza, con afán de mejorar sus condiciones de vida, que mantienen una actitud vinculada a lo positivo, entre otros; son los que adhieren fundamento a esta percepción de deseabilidad. Y precisamente, estas personas de enorme capacidad de reponerse a la adversidad extrema son casos de personas que no han caído en este trastorno aquí propuesto.

Con respecto a la enfermedad de la diabetes, a pesar de que, como lo habíamos mencionado anteriormente, al menos varios de sus factores de riesgo se podrían controlar voluntariamente, no existe o no se conoce que se le atribuya causalidad interna, individual o personal a su padecimiento. Nadie otorga deseabilidad de esta enfermedad a quien la padece. Al menos, no es de uso frecuente la expresión: "Tiene diabetes porque quiere".

Ahora veamos el tratamiento cuando la enfermedad ya está instalada. En el caso de la diabetes, es posible el tratamiento médico (básicamente administración de insulina), dieta y actividad física. Es decir, es posible restaurar la salud siguiendo el tratamiento. Así, disminuyen o desaparecen los síntomas y signos, tales como la constante necesidad de orinar, sed inusual, visión borrosa, infecciones frecuentes, etc.

En el caso del TRAPSIPO, ¿cuál sería el tratamiento? ¿Qué medidas/estrategias se podrían tomar para disminuir o erradicar la sintomatología del TRAPSIPO? ¿Hay una fórmula para combatir el conformismo, fatalismo, negativismo, añoranza por el pasado, pereza, desesperanza, falta de vitalidad y de esfuerzo, carencia de iniciativa, baja autoestima, desvalorización y otros síntomas? La dimensión de las interrogantes nos lleva automáticamente a ver el principal factor de riesgo; la pobreza, que está generando la aparición de este trastorno. Incidiendo en su disminución, el número de personas que caen en el TRAPSIPO también decrecería.

Es obvio el carácter complejo del TRAPSIPO. No es tan sencillo como la enfermedad de la diabetes. Y la respuesta es puntual, incluso está en las iniciales dentro de su denominación: TRAPSIPO (Trastorno Psicológico de la Pobreza); dicha respuesta consiste en erradicar la pobreza, la inequidad y la desigualdad. Por ello, se conjetura que en las sociedades más equitativas en la que sus habitantes gozan de mayor estándar de vida ver ciudadanos conformistas, fatalistas, con baja autoestima, con desvalorización, etc., estaría en su mínima expresión; si los hay, otras razones estarían presentes y no la carencia económica. Y la razón recae en que los factores de riesgo, que en el Perú son manifiestos, en ellas no existen. $\mathrm{O}$ al menos están reducidos a su mínima expresión: pobreza casi inexistente, lo mismo que en la deserción escolar, el trabajo infantil, etc. Uno que otro factor de los mencionados tal vez estaría presente, pero no sería la norma.

Por otro lado, todo cabe indicar que quienes sufren de TRAPSIPO experimentan continuamente emociones displacenteras que afectan su bienestar; la preocupación, la ira y frustración son constantes. Así como también la tristeza, el rencor y la vergüenza. La amargura por no contar con un sueldo fijo, la tristeza de no haber estudiado una profesión, la cólera de no poder salir a comer afuera con la familia, la impotencia de no poder comprar juguetes a los hijos, el rencor a la gente que tiene recursos, la sensación de vergüenza y de humillación por vivir en una casa modesta, etc. Al respecto, expresan su sensación, por ejemplo, de no ser escuchados ni atendidos debidamente en los servicios públicos por carecer de educación y por la condición de precariedad económica en la que se encuentran. Y lo que es más, en muchas ocasiones su argumento transporta la sensación de que "a los que tienen les gusta humillar".

En tal sentido, es muy posible que esta emocionalidad, junto a las actitudes referidas anteriormente; apatía, desidia, conformidad, resignación, entre otras; puedan impedir que políticas públicas diseñadas para combatir la pobreza tengan resultados favorables. En esta dirección, Zavaleta (2011), quien se ocupa de aquellas dimensiones de la pobreza que generalmente no están en las estadísticas nacionales ni mundiales como el sentimiento de vergüenza y humillación, afirma que las personas en situación de pobreza frecuentemente experimentan estos sentimientos que no sólo afectan la salud emocional y física, sino que también se constituyen en un obstáculo para vivir en mejores condiciones de vida.

Al respecto, por ejemplo, si alguien decide no asistir a una posta médica por anticipar que en ese establecimiento será discriminado, este hecho puede influir en que no tome acciones para mejorar sus condiciones de salud. O, en otro ejemplo, si una 
persona siente vergüenza porque todos "la miran" por llevar la ropa gastada o raída, esa persona no acudirá al centro educativo en el que estudian sus hijos, o a pedir un crédito o buscar empleo.

"Cuando tú te vas a la posta, asistes pe', las enfermeras a veces te tratan mal a veces, pero cuando ya tienes una profesión tú haces con tu plata ya lo que quieras y allí nadie te está diciendo un montón de cosas. A veces, esteeee cuando yo estaba embarazada de mi hijo, me hacía mi control, no te quieren atender, por más que estés con dolor, no te quieren atender, te ponen a un lado por más que estés con fiebre, no te hacen caso, no te dan buena atención. Cuando ya te ven en las últimas allí recién te hacen caso, sino, no te hacen caso. Yo digo que será por eso, porque no tenemos estudios, de repente, porque a veces nos ven nos ven pobres, ellos ya al tener esa profesión ya se creen ver mucho más que nosotros". Grupo Focal, mujeres de 18 a 29 años [Mujer de 19 años] Pucallpa (Morales, 2020).

\section{Implicaciones de esta nueva categoría nosológica}

La propuesta de la existencia del TRAPSIPO, lleva consigo que nos despojemos de la idea de que quienes lo sufren son básicamente los responsables de las condiciones en las que viven. La pobreza genera actitudes y conductas que reproducen la pobreza. La aceptación del TRAPSIPO como una nueva categoría nosológica implicaría, más bien, considerar a estas personas como víctimas de un modelo de sociedad que actúa y ejerce su rol protagónico en el surgimiento de psicopatologías; el TRAPSIPO, precisamente, sería consecuencia de ello. Creemos que es hora de exponerlo a la luz, de reconocer que sí existe, sólo faltaba escudriñar la realidad y asignarle una denominación.

Reflexionar con cierta profundidad acerca de este punto es de suma importancia porque aborda el factor psicológico involucrado en la perpetuación de la pobreza pero que es generado por la propia pobreza. Al respecto, lo aseverado por Castillo y Rivera-Gutiérrez (2018) cobra notable sentido: en la medida que los pobres son considerados responsables de su situación (i.e., atribución interna), sería menos probable que exista cualquier intención o motivación por modificar los condicionantes que subyacen a la existencia de la pobreza. Los posibles impactos de esta forma de explicar el origen de la pobreza se reflejan en las actitudes hacia ricos y pobres (Kluegel et al, 1995; Sigelman, 2012; citados por Castillo \& Rivera-
Gutiérrez, 2018), el grado en que las desigualdades de ingreso se perciben como justas (Schneider \& Castillo, 2015; citados por Castillo \& Rivera-Gutiérrez, 2018), y la percepción general de las desigualdades sociales (Bullock \& Limbert, 2003; citados por Castillo \& Rivera-Gutiérrez, 2018).

Ciertamente, postular esta nueva categoría diagnóstica pondría coto a expresiones y adjetivos que dañan y ofenden a las personas que están inmersas en ella. Es decir, las consideraciones que los pobres son perezosos, irresponsables, conformistas y carentes de espíritu emprendedor (Addison et al., 2008; Dakduk et al., 2010) (características que son en realidad signos del TRAPSIPO), persistirán a no ser que esta propuesta sea aceptada por la comunidad científica.

Y lo más relevante, el diseño de políticas públicas e intervenciones para mejorar la calidad de vida de los pobladores que viven en condiciones de pobreza podría beneficiarse con esta nueva propuesta nosológica. El reconocimiento de este trastorno permitiría justificar la realización de intervenciones psicológicas en individuos que manifiesten las actitudes y conductas que lo caracterizan, con el fin de modificarlas. Con ello, no solamente se establecerían mejores niveles de bienestar psicológico en esa población, sino que también se contribuiría a que logren superar actitudes y conductas que constituyen un impedimento para que las políticas públicas diseñadas para combatir la pobreza tengan mejores resultados, e incluso para que los propios individuos asuman de manera autónoma acciones más eficaces para mejorar su calidad de vida.

Declaración de conflicto de interés: Declaro que el presente artículo no presenta conflicto de intereses $\mathrm{y}$, asimismo, cumple con las normas éticas en su desarrollo.

\section{Correspondencia:}

Luis Hesneide Morales López

Correo electrónico: luis.morales.1@upch.pe

\section{REFERENCIAS}

Addison, T., Harper, C., Prowse, M. P., Shepherd, A., Barrientos, A., Braunholtz-Speight, T., Evans, A., Grant, U., Hickey, S., Hulme, D., \& Moore, K. (2008). The Chronic poverty report 2008/09: Escaping poverty traps. Chronic Poverty Research Centre.

Alarcón, R. (1986). Psicología, pobreza y subdesarrollo. 
Instituto Nacional de Investigación y Desarrollo de la Educación.

American Diabetes Association (s/f). Learn the genetics of diabetes. https:/www.diabetes.org/diabetes/ genetics-diabetes

Ardila, R. (1979). Psicología social de la pobreza. En J. O. Whittaker (Ed.). La psicología social en el mundo de hoy (pp. 399-418). Trillas.

Babcock, E. (2014). Using brain science to design new pathways out of poverty. Crittenton Women's Union.

Bauman, Z. (2003). Wasted lives: Modernity and its outcasts. Wiley-Polity.

Castillo, J. C. \& Rivera-Gutiérrez (2018). Dimensiones comunes a las atribuciones de pobreza y riqueza. Psykhe, 27(2), 1-10. http://dx.doi.org/10.7764/ psykhe.27.2.1152

Dakduk, S., González, M., \& Malavé, J. (2010). Percepciones acerca de los pobres y la pobreza: Una revisión. Revista Latinoamericana de Psicología, 42(3), 413-425.

De Gaulejac, V. (2015). Las fuentes de la vergüenza. Sapere Aude.

Hammen, C. (2005). Stress and depression. Annual Review of Clinical Psychology, 1, 293-319. https:// doi.org/10.1146/annurev.clinpsy.1.102803.143938

Kandel, E. R. (2005). Psychiatry, psychoanalysis, and the new biology of mind. American Psychiatric Publishing, Inc.

Lera, M., J. (2009). Impacto de la pobreza crónica en la resiliencia; claves para entender la perpetuidad de la miseria. Paideia, 46, 73-97.

Lewis, O. (1972). La cultura de la pobreza. Anagrama. Mathewson, T. G. (2017). The Atlantic Monthly: How Poverty Changes the Brain. Economic Mobility Pathways.https://www.empathways.org/news/article/ the-atlantic-monthly-how-poverty-changes-the-brain Medina, I., \& Florido, A. L. (2005). La pobreza desde los pobres. Un estudio de caso en Guadalajara. Libros en Red.

Merz, E.C., Wiltshire, C.A., \& Noble, K. (2019). Socioeconomic inequality and the developing brain: Spotlight on language and executive function. Child Development Perspectives, 13, 15-20. https:// doi.org/10.1111/cdep.12305

Morales, L. H. (2020). Expectativas de futuro en pobladores que viven en situación de pobreza de tres asentamientos humanos en Chimbote, Puno y Pucallpa . [Tesis de doctorado en psicología, Universidad Peruana Cayetano Heredia]. Repositorio Institucional de la Universidad Peruana Cayetano Heredia. http://repositorio.upch.edu.pe/bitstream/ handle/upch/8613/Expectativas_MoralesLopez_ Luis.pdf? s e que n c e $=1 \&$ is A 11 ow e d $=\bar{y}$ Organización Mundial de la Salud (2019). CIE11. Clasificación Internacional de Enfermedades. Organización Mundial de la Salud. https://icd.who. int/es

Organización Mundial de la Salud. (2018). Diabetes. World Health Organization. https:/www.who.int/es/ news-room/fact-sheets/detail/diabetes

Rocheleau, J. (2019). How poverty shapes a child's mind and brain. https://www.brainfacts.org/neurosciencein-society/law-economics-and-ethics /2019/howpoverty-shapes-a-childs-mind-and-brain-101419

Rodríguez, C. (1989). Cicatrices de la pobreza. Nueva Sociedad.

Seligman, M. E. P. (1975). Helplessness: On depression, development, and death. W. H. Freeman.

Sen, A. (2000). Desarrollo y libertad. Planeta.

Zavaleta, D. (2011). Pobreza, vergüenza y humillación: una propuesta de medición. Revista Latinoamericana de Desarrollo Humano, 76, 1-7. https://www.ophi. org.uk/wp-content/uploads/Verguenza1.pdf 